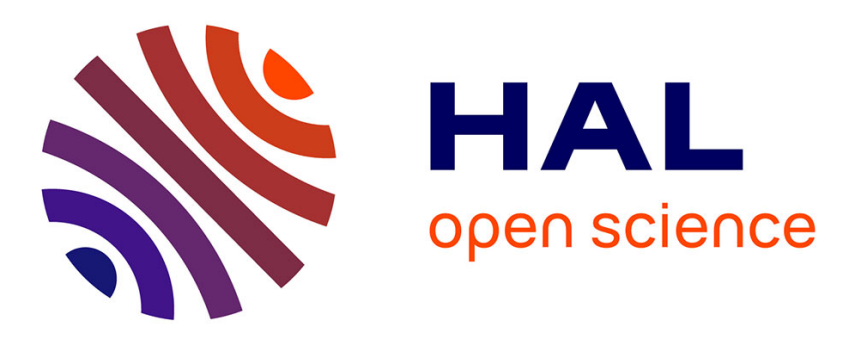

\title{
Préparation par électrolyse à basse température d'hydrures de Palladium
}

B. Souffaché, D.S. Maclachlan, J.P. Burger

\section{To cite this version:}

B. Souffaché, D.S. Maclachlan, J.P. Burger. Préparation par électrolyse à basse température d'hydrures de Palladium. Revue de Physique Appliquée, 1979, 14 (8), pp.749-756. 10.1051/rphysap:01979001408074900 . jpa-00244656

\section{HAL Id: jpa-00244656 https://hal.science/jpa-00244656}

Submitted on 1 Jan 1979

HAL is a multi-disciplinary open access archive for the deposit and dissemination of scientific research documents, whether they are published or not. The documents may come from teaching and research institutions in France or abroad, or from public or private research centers.
L'archive ouverte pluridisciplinaire HAL, est destinée au dépôt et à la diffusion de documents scientifiques de niveau recherche, publiés ou non, émanant des établissements d'enseignement et de recherche français ou étrangers, des laboratoires publics ou privés. 


\title{
Préparation par électrolyse à basse température d'hydrures de Palladium
}

\author{
B. Souffaché, D. S. MacLachlan $\left({ }^{*}\right)$ et J. P. Burger \\ Laboratoire de Physique des Solides, Université Paris-Sud, 91405 Orsay
}

(Reçu le 24 février 1979, révisé le 7 mai 1979, accepté le 7 mai 1979)

\begin{abstract}
Résumé. - Les propriétés des hydrures de Pd non stœchiométriques mais stables de formule $\mathrm{PdH}_{x}$ avec $x<0,7$ sont bien connues; nous décrivons dans ce travail une méthode de préparation d'hydrures plus concentrés, $y$ compris le composé stœchiométrique $\mathrm{PdH}$; la préparation ise fait par électrolyse à basse température $\left(-80^{\circ} \mathrm{C}\right)$ dans un bain composé d'alcool éthylique et d'acide sulfurique; les hydrures préparés dans ces conditions sont instables à température ambiante et ne peuvent être manipulés qu'en dessous de $-80^{\circ} \mathrm{C}$; nous décrivons quelques propriétés de ces hydrures et nous discutons l'influence d'une variation continue de $x$ sur les propriétés d'alliages magnétiques à base de $\mathrm{PdH}_{x}$.

Abstract. - The properties of stable non stoechiometric $\mathrm{PdH}_{x}$ hydrides with $x<0.7$ are well-known; we describe here a method of preparation for more concentrated hydrides, including the stoechiometric PdH compound; the preparation is done at low temperature $\left(-80^{\circ} \mathrm{C}\right)$ by electrolysis using an alcohol + acid bath; the as prepared hydrides are unstable at room temperature and can only be handled below $-80^{\circ} \mathrm{C}$; we describe some of the properties of these hydrides and discuss the influence of a continuous change of $x$ on the physical properties of $\mathrm{PdH}_{x}$ based magnetic alloys.
\end{abstract}

L'étude des propriétés physiques d'hydrures métalliques connaît ces dernières années un très net regain de faveur lié à l'importance qu'ont ces composés dans différents domaines appliqués (fragilisation des métaux, stockage de l'hydrogène...) et fondamentaux (observation de diagrammes de phases du type gazliquide, possibilité d'études, d'effets isotopiques et anharmoniques, diffusion non classique, supraconductivité liée aux vibrations de l'hydrogène,...). Des progrès ont également été réalisés dans les calculs de structure électronique, qui laissent apparaître des aspects nouveaux permettant de mieux cerner le problème de la stabilité de ces hydrures.

Il apparaît que, parmi les nombreux hydrures métalliques, les plus étudiés soient ceux à base de Pd, de formule $\mathrm{PdH}_{x}$; les efforts expérimentaux [1] ont porté essentiellement, jusqu'à ce jour, sur les hydrures non stœchiométriques, avec $x<0,7$ qui sont réputés stables à température ambiante et dont les propriétés s'interprètent qualitativement par le modèle des bandes rigides où chaque hydrogène transfère son électron à la bande $4 \mathrm{~d}$ du $\mathrm{Pd}$ qui se remplit progressivement; il est curieux de constater qu'une extension relativement faible du domaine de concen-

$\left(^{*}\right)$ Adresse permanente : Physics Department, University of the Witwatersrand, Johannesburg, Afrique du Sud. tration en hydrogène (de $x \simeq 0,7$ jusque vers $x=1$ ) a permis de mettre en lumière de nouvelles propriétés comme par exemple l'apparition de supraconductivité ; ces hydrures plus concentrés sont malheureusement instables à température ambiante et leur manipulation est donc difficile.

Le but de ce travail est de montrer comment on peut préparer de tels hydrures instables. Dans un premier chapitre, nous donnerons des rappels sur la structure atomique et électronique des hydrures de $\mathrm{Pd}$; dans le deuxième nous tenterons de justifier l'intérêt qu'il y a à préparer de tels hydrures; le chapitre suivant décrira en détail la méthode de préparation employée et dans un dernier chapitre nous donnerons quelques résultats qui montrent l'importance du domaine $0,7<x<1$.

1. Rappels sur la structure atomique et électronique de $\mathrm{PdH}_{x^{*}}-\mathrm{La}$ formation spontanée d'hydrures de $\mathbf{P d}$ est un fait connu depuis fort longtemps (pour une revue, voir référence [1]). On peut très schématiquement caractériser leurs propriétés par les points suivants : 1) le système $\mathrm{Pd}+\mathrm{H}$ présente un diagramme de phase particulièrement simple ressemblant à celui d'une transition gaz $\rightarrow$ liquide avec une précipitation en deux phases $\alpha$ (ou phase gazeuse) et $\beta$ (ou phase liquide) en dessous d'une température 
et d'une pression critiques ; 2) il existe une tendance à la formation d'hydrures non stœchiométriques avec $x \simeq 0,65$ (limite de la phase $\alpha \rightarrow \beta$ quand la température tend vers zéro), 3) la structure électronique de $\mathrm{PdH}_{x}$ est très différente de celle $\mathrm{du} \mathrm{Pd}$.

1.1 Diagramme DE PHASE ET STRUCTURE CRISTALLINE. - La figure 1 montre le diagramme de phase bien connu [1] du système $\mathrm{Pd}+\mathrm{H}$. On peut distinguer pour $T<T_{\mathrm{c}}$ trois domaines de concentration $x<x_{\alpha}(T), x>x_{\beta}(T)$ et $x_{\alpha}(T)<x<x_{\beta}(T)$. Le premier domaine représente la phase gaz de réseau, le troisième la phase liquide et, dans le domaine intermédiaire, on a coexistence des deux phases $\alpha$ et $\beta$. L'observation de la diffusion de neutrons [3] et les mesures de R.M.N. [3] permettent par ailleurs d'affirmer que les atomes d'hydrogène occupent les sites octaédriques du palladium et qu'ils diffusent rapidement d'un site octaédrique à l'autre, du moins tant que la température est supérieure à environ $140 \mathrm{~K}$. Comme il y a exactement un site interstitiel de nature octaédrique par atome de $\mathrm{Pd}$, il y a possibilité de former le composé stœchiométrique $\mathrm{PdH}$.

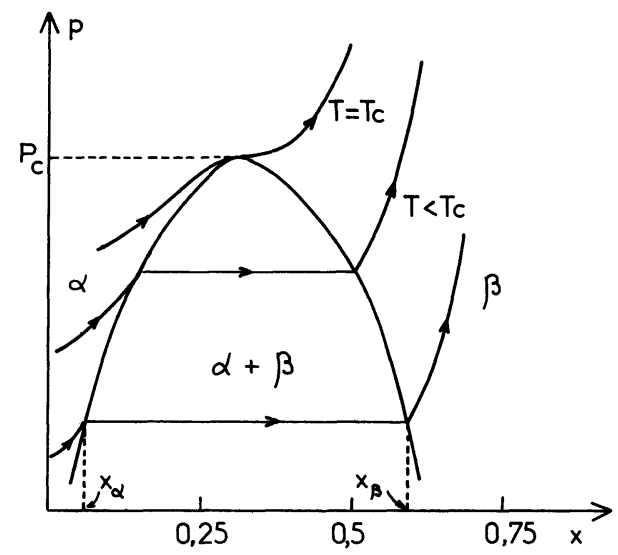

Fig. 1. - Schéma du diagramme de phase de $\mathrm{PdH}_{x}$.

[Schematic phase diagram of $\mathrm{PdH}_{x}$.]

L'origine des interactions $\mathbf{H}-\mathbf{H}$ qui conduit au diagramme de phase ci-dessus n'est pas encore bien élucidée ; il est possible qu'elle soit en partie de nature élastique via le champ de dilatation entourant chaque hydrogène [4], il est possible également que des interactions de plus courte portée, de nature covalente, soient importantes [5]. Un fait significatif à remarquer est que $x_{\beta}(T \rightarrow 0) \simeq 0,65$ alors qu'un modèle simple de gaz de réseau avec une interaction régulière en fonction de $x$ conduirait invariablement à

$$
x_{\beta}(T \rightarrow 0)=1 \text {, }
$$

c'est-à-dire l'occupation de tous les sites octaédriques dans la limite $T=0$. Cette observation indique une stabilité particulière du composé non stœchiométrique $\mathrm{PdH}_{0,65}$ et explique qualitativement la difficulté à préparer des hydrures plus concentrés. D'ailleurs, la plupart des propriétés physiques de $\mathrm{PdH}_{x}$ montrent des anomalies pour cette concentration particulière $x \simeq 0,65[1]$.

1.2 StruCtURE ÉleCtRoniQUe. - Les propriétés électroniques de $\mathrm{PdH}_{x}$ ont été interprétées dans le cadre du modèle des bandes rigides où on admet que chaque hydrogène apporte un électron à la bande $4 d+5 s-p$ du palladium, dont le niveau de Fermi se déplace continuellement vers les hautes énergies. Ce modèle conduit avant tout à un remplissage progressif des 0,36 trois/atomes que possède la bande $4 d$ du Pd. On sait que le niveau de Fermi $\varepsilon_{\mathrm{F}}$ se trouve dans le Pd pur au voisinage immédiat d'un pic de densité d'états qui donne d'ailleurs à ce métal des propriétés tout à fait particulières (forte valeur de la chaleur spécifique électronique $\gamma$, susceptibilité de Pauli $\chi$ élevée, tendance au ferromagnétisme, absence de supraconductivité...). Le remplissage de la bande $4 d$ se traduit par de fortes diminutions de $\gamma$ [6] et $\chi$ [7] en accord avec ce modèle des bandes rigides. Si on continue d'apporter des électrons, alors que le remplissage de la bande $4 d$ est terminé, ceux-ci ne pourront qu'occuper des états s-p dont le spectre d'énergie recouvre et prolonge celui de cette bande $4 \mathrm{~d}$ (Fig. 2). Ces hydrures, plus concentrés, devraient ressembler d'une certaine façon aux métaux nobles tels Ag, ce qui est bien le cas. Mais le modèle de bande rigide présente cependant deux défauts : d'une part, il n'explique en rien la stabilité des hydrures, c'est-à-dire le fait que la formation de $\mathrm{Pd}-\mathrm{H}_{x}$ (avec $x \sim 0,65$ ) se fait spontanément avec gain d'énergie, et, d'autre part, on ne comprend pas pourquoi il faut 0,65 électron pour remplir la bande $d$ alors qu'il n'y a que 0,36 trou dans celle-ci. Des calculs plus récents [5], [8] montrent en fait que le potentiel nucléaire du proton déplace et déforme considérablement les bandes énergétiques du $\mathrm{Pd}$, d'où des écarts très sensibles au modèle des bandes rigides. Pour bien comprendre l'effet de ce potentiel, il faut savoir où se trouvent dans le Pd les états qui ont la bonne symétrie $1 \mathrm{~s}$ autour du site de l'hydrogène : ce sont en effet ces

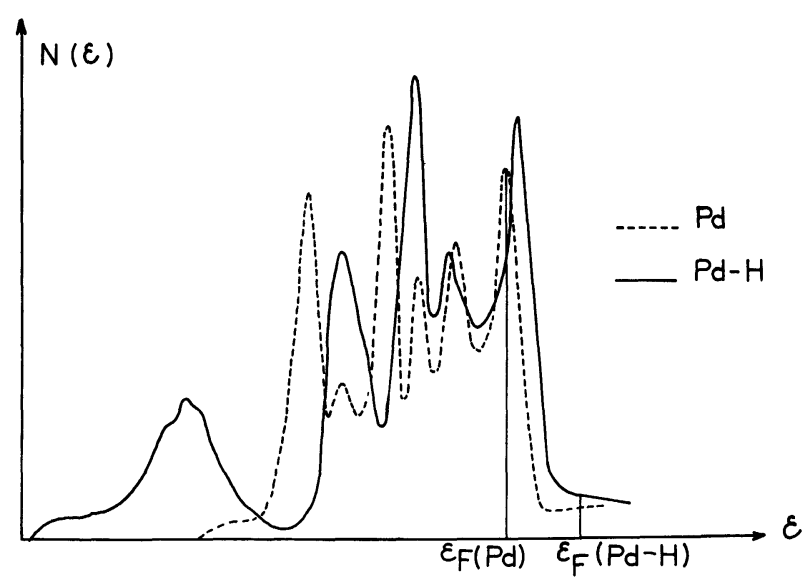

Fig. 2. - Densité d'état de Pd en Pd-H (voir références [8] et [20]).

[Density of states of Pd and Pd-H (see references [8] and [20]).] 
états qui seront les plus perturbés et qui verront leur énergie abaissée. Il se forme ainsi une bande d'énergie juste en dessous de la bande 4d (voir Fig. 2) qu'on appelle la bande de l'hydrogène et pour la présence de laquelle il existe maintenant des preuves expérimentales [9]. C'est cette bande qui est responsable de la stabilité des hydrures [10]. Il y a également des modifications de la structure de bande au voisinage du niveau de Fermi $\varepsilon_{\mathrm{F}}$. Des états vides dans le Pd (car au-dessus de $\varepsilon_{\mathrm{F}}$ ) passent en dessous de $\varepsilon_{\mathrm{F}}$. L'électron apporté par l'hydrogène entre préférentiellement dans ces états avant d'occuper les états vides immédiatement au-dessus de $\varepsilon_{\mathrm{F}}$, ce qui a pour effet de déplacer le niveau de Fermi vers le haut. La contribution positive à l'énergie de $\mathrm{PdH}_{x}$, liée au déplacement vers le haut de $\varepsilon_{\mathrm{F}}$, reste faible tant que le niveau de Fermi se trouve dans la bande $4 \mathrm{~d}$ : la forte valeur de la densité d'états $N\left(\varepsilon_{\mathrm{F}}\right)$ fait que $\varepsilon_{\mathrm{F}}$ n'augmente que très lentement avec $x$; mais il n'en est plus de même dès que $\varepsilon_{\mathrm{F}}$ passe dans la bande $5 \mathrm{~s}$-p c'est-à-dire pour $x>0,65$ : la faible densité d'états $N\left(\varepsilon_{\mathrm{F}}\right)$ fait maintenant que $\varepsilon_{\mathrm{F}}$ augmente rapidement avec $x$, ce qui est très défavorable à la stabilité d'ensemble de l'hydrure. On peut expliquer ainsi la tendance à l'instabilité des hydrures dès que $x$ dépasse la valeur 0,65 .

\section{Intérêt de préparer des hydrures plus concentrés. -}

Nous venons de voir que l'hydrure non stœechiométrique $\mathrm{PdH}_{0,65}$ présente une stabilité particulière liée vraisemblablement au changement de nature des niveaux qui peuvent accepter l'électron de l'hydrogène dès que $x$ dépasse 0,65 . Il s'ensuit que les hydrures plus concentrés ont été moins étudiés tout simplement parce qu'ils montrent une tendance à l'instabilité qui grandit au fur et à mesure qu'on essaie de s'approcher de $x=1$; on pensait également qu'ils n'avaient qu'un intérêt limité dans la mesure où leur structure électronique ressemble fort à celle des métaux nobles. Cependant, plusieurs faits motivent actuellement le regain d'intérêt porté sur les concentrations $x$ plus élevées :

- Il est possible dans le système $\mathrm{PdH}_{x}$ de faire varier de manière continue le nombre d'électrons de conduction, et donc aussi la densité d'états $N\left(\varepsilon_{\mathrm{F}}\right)$ au niveau de Fermi rien qu'en modifiant le paramètre $x$. Cette situation ressemble à celle qu'on peut obtenir avec une série d'alliages fabriqués à partir de métaux purs de valence différente (par exemple $\mathrm{CuZn}$ ), avec l'avantage dans le cas présent qu'on peut faire varier cette densité électronique en utilisant un seul échantillon de Pd. Très schématiquement, on peut estimer que le nombre d'électrons de conduction au-dessus de la bande $4 d$ pleine est proportionnel, sinon égal, à $x$ : la plage de densité électronique qu'on peut ainsi couvrir, typiquement de 0,65 à 1 électron de conduction par atome de Pd, est certes faible, mais de nombreuses propriétés physiques dépendent fortement de la densité d'états $N\left(\varepsilon_{\mathrm{F}}\right)$, surtout si on étend ces considérations à celles des alliages dilués
$\operatorname{Pd}_{1-y} M_{y} H_{x}$ où $M$ est une impureté magnétique $(\mathbf{M}=\mathrm{Cr}, \mathbf{M n}, \mathrm{Gd} . .$.$) : on peut étudier l'effet Kondo,$ la relaxation de Korringa, les interactions indirectes du type Rudermann-Kittel entre impuretés en fonction de $N\left(\varepsilon_{\mathrm{F}}\right)$, etc... Un fait important découlant des calculs théoriques [8] est qu'il n'est pas justifié de considérer seulement la densité d'états globale $N\left(\varepsilon_{\mathrm{F}}\right)$, mais qu'il faut décomposer celle-ci en ses composantes locales sur les sites $\mathrm{Pd}$ et $\mathrm{H}$; les modèles théoriques indiquent que celles-ci varient en sens inverse, diminuant avec $x$ sur le site $\mathrm{Pd}$ mais augmentant sur le site $\mathrm{H}$ : des mesures locales (par relaxation de Korringa par exemple) doivent être capables de confirmer ces points.

- L'apparition de supraconductivité pour $x>0,7$ : elle est caractérisée par des $T_{\mathrm{c}}$ relativement élevées $\left(T_{\mathrm{c}} \leqslant 10 \mathrm{~K}\right.$ pour $\left.x=1\right)$ et qui varient fortement avec $x\left(T_{\mathrm{c}} \simeq 0,1 \mathrm{~K}\right.$ pour $\left.x \simeq 0,7\right)$. Cette observation montre, à elle toute seule, l'intérêt qu'il peut y avoir à contrôler ce domaine de concentration. Cette supraconductivité pose immédiatement le problème de son origine, vu que les métaux monovalents sont connus pour ne pas être supraconducteurs. Le fait que $T_{\mathrm{c}}$ varie quand on remplace $\mathrm{H}$ par son isotope $\mathrm{D}$ montre bien que les vibrations optiques de $\mathrm{H}$ (ou D) sont impliquées dans ce phénomène.

- On constate également que l'adjonction d'impuretés substitutionnelles du Pd augmente en général fortement l'instabilité des hydrures; les concentrations maxima d'hydrogène absorbé diminuent en effet fortement avec la concentration d'un certain nombre d'impuretés; une étude systématique du domaine de stabilité devrait aboutir à une meilleure compréhension des facteurs qui conditionnent la formation et la stabilité des hydrures métalliques.

\section{Préparation d'hydrures concentrés par électrolyse} à basse température. - Avant de décrire en détail notre méthode de préparation, nous allons rappeler brièvement les méthodes utilisées à ce jour pour couvrir la gamme de concentration $x<0,7$ ainsi que les différents essais tentés pour obtenir des concentrations plus élevées. Trois techniques ont été employées à ce jour : absorption directe à partir d'une atmosphère $\mathrm{d}$ 'hydrogène sous pression [1], [11], [12], électrolyse [1] et, plus récemment, implantation [13]. La première méthode, la plus employée, a l'avantage de conduire à des situations d'équilibre thermodynamique caractérisées par une relation $x=f(p)$ où $p$ est la pression d'hydrogène appliquée. Le tableau suivant donne une idée de l'échelle de pression nécessaire pour atteindre des concentrations $x$ (en phase $\beta$ ) données [14] :

$\begin{array}{cccc}p & x & x & x \\ \text { (atmosphère) } & \left(\grave{a} 50^{\circ} \mathrm{C}\right) & \left(\mathrm{à} 0^{\circ} \mathrm{C}\right) & \left(\mathrm{a}-78^{\circ} \mathrm{C}\right) \\ - & - & - & - \\ 10^{-3} & \text { phase } \alpha & \text { phase } \alpha & 0,73 \\ 1 & 0,66 & 0,73 & 0,82 \\ 10^{3} & 0,83 & 0,85 & 0,91\end{array}$


Ce tableau laisse apparaître la difficulté à dépasser la valeur $x \simeq 0,9$ pour des pressions raisonnables d'hydrogène même si on abaisse la température d'absorption. D'autres désavantages de cette méthode sont liés à la cinétique d'absorption qui est contrôlée par l'état de la surface où se produit la dissociation des molécules $\mathrm{H}_{2}$ : l'expérience montre que seuls des échantillons présentant de grands rapports surface sur volume (poudres, films minces) s'hydrogènent rapidement à suffisamment basse température. On peut certes augmenter la cinétique en chauffant les échantillons mais on perd alors rapidement sur la quantité d'hydrogène absorbée pour une pression d'hydrogène donnée. Il s'avère donc pratiquement impossible par cette méthode d'hydrogéner au-delà de $x \simeq 0,7$ des échantillons massifs ayant environ $100 \mu$ d'épaisseur; or, la mesure de la susceptibilité magnétique par exemple nécessite précisément de tels échantillons; d'autre part, la présence de la cellule de pression rend l'accès aux échantillons fort malaisé, d'où des difficultés de manipulation dès qu'on veut transférer ceux-ci dans un autre appareil de mesure.

La technique d'implantation à basse température de films minces de Pd par des ions $\mathrm{H}^{+}$ou $\mathrm{D}^{+}$est d'un emploi beaucoup plus récent. Elle présente l'avantage de couvrir sans difficulté toute la gamme de concentration $x$ désirée. Mis à part le fait que très peu de laboratoires disposent de l'équipement nécessaire, il nous faut signaler deux désavantages de cette méthode : 1) il est difficile d'obtenir des profils de concentration homogènes, 2) le bombardement ionique introduit de nombreux défauts structuraux (atomes déplacés de leur position d'équilibre, lacunes...). Les échantillons ainsi préparés ont presque toujours des résistivités résiduelles très élevées (supérieures à $10 \mu \Omega \mathrm{cm}$ ) même en ce qui concerne le composé stœchiométrique $\mathrm{PdH}$. L'introduction d'hydrogène dans le Pd par électrolyse est également largement employée. Cette méthode se caractérise par une grande simplicité de mise en œuvre : les densités de courant nécessaires pour atteindre $x \simeq 0,7$ sont très faibles et la méthode est opérante aussi bien pour des films minces que pour des échantillons massifs ; le problème de l'état de surface est en effet beaucoup moins important vu qu'on absorbe directement des ions $\mathrm{H}^{+}$. Peu d'essais [15], [16] ont été faits à ce jour pour dépasser des concentrations de l'ordre de $x \sim 0,8$, les essais préliminaires ayant montré que les densités de courant nécessaires devenaient vite prohibitives. Cette limitation est en grande partie liée à la température de l'électrolyse qui s'effectue pour des raisons évidentes au voisinage de l'ambiante.

3.1 Electrolyse a BASSE TEMPÉRATURE. - Les rares données existant dans la littérature [14] indiquent une forte diminution des pressions d'hydrogène $p(x)$ nécessaires pour atteindre une concentration $x$ donnée si on abaisse la température d'hydrogénation en dessous de la température ambiante. Le tableau précédent indique que dans le domaine $x \sim 0,7-0,9$ on gagne pratiquement un facteur mille sur les pressions quand on passe de $0^{\circ} \mathrm{C}$ à $-78^{\circ} \mathrm{C}$, soit un gain fort appréciable. On peut penser qu'il en est de même pour l'électrolyse, d'où l'idée d'abaisser la température de fonctionnement de celle-ci. Il y a cependant deux limitations dans cette course aux basses températures, liées à la mobilité des ions $\mathrm{H}^{+}$dans l'électrolyte et dans le palladium. Ces deux points seront analysés ci-dessous.

3.1.1 Le bain d'électrolyse. - La nécessité de disposer d'un bain riche en ions $\mathrm{H}^{+}$et suffisamment conducteur à $T \simeq-80^{\circ} \mathrm{C}$ nous a fait adopter pour électrolyte un mélange d'éthanol absolu pur $\mathrm{C}_{2} \mathrm{H}_{5} \mathrm{OH}$ et d'acide sulfurique $\mathrm{SO}_{4} \mathrm{H}_{2}$. La proportion d'acide sulfurique utilisée est de l'ordre de $10 \%$ en volume. Ce mélange ne se solidifie pas avant $-110^{\circ} \mathrm{C}$ et possède à la température ambiante une résistivité électrique de l'ordre de $20 \Omega \mathrm{cm}$. Cette résistivité électrique augmente d'un facteur cinq quand on abaisse la température vers $-80^{\circ} \mathrm{C}$. La cellule d'électrolyse est placée dans un bac contenant un mélange de glace carbonique et d'acétone qui assure la température de $-80^{\circ} \mathrm{C}$. Ce bac est lui-même placé dans un dewar, ce qui permet de maintenir la température du bain réfrigérant avec une autonomie de l'ordre de $15 \mathrm{~h}$.

3.1.2 Les échantillons de Pd. - Les échantillons utilisés se présentent sous forme de rubans d'épaisseur de l'ordre de 50 à $100 \mu$, de 5 à $10 \mathrm{~mm}$ de large et de plusieurs centimètres de long. Ils sont obtenus par laminage d'un lingot fondu au four à induction. L'épaisseur a été choisie pour permettre une bonne diffusion de l'hydrogène à travers toute l'épaisseur des rubans en un temps raisonnable : en effet, la constante de diffusion $D$ à $-80^{\circ} \mathrm{C}$ est de l'ordre de $10^{-9} \mathrm{~cm}^{2} / \mathrm{s}$, d'où un temps de diffusion $t \simeq e^{2} / D$ de l'ordre d'une dizaine d'heures. Il est clair que de tels échantillons massifs représentent un cas beaucoup moins favorable que celui de films minces qui peuvent être hydrogénés beaucoup plus rapidement suivant la même technique. Le ruban de Pd forme une des électrodes du circuit d'électrolyse, l'autre électrode étant formée d'un ruban de platine.

\subsubsection{Contrôle du processus d'hydrogénation. -} Nous mesurons, durant l'électrolyse, la résistivité des rubans de Pd afin de suivre et contrôler le processus d'absorption d'hydrogène; à chaque extrémité du ruban de $\mathrm{Pd}$, sont soudés deux fils de $\mathrm{Cu}$, ce qui permet de mesurer sa résistance par une méthode à quatre points; ces deux extrémités sont insérées dans deux types de verre creux et noyées dans une résine époxy afin d'isoler les quatre fils de cuivre du bain d'électrolyse. Un rupteur séquentiel interromp périodiquement le courant d'électrolyse $I_{E}$ et envoie dans le ruban de Pd un courant de mesure $I_{R}$ foumi par une alimentation stabilisée en courant; la différence de potentiel apparaissant aux bornes du ruban 
est enregistrée sur un traceur automatique $\left(V_{\mathrm{R}}, t\right)$. Pour une hydrogénation qui dure environ $24 \mathrm{~h}$, nous mesurons $V_{R}$ toutes les 6 min., la durée de la mesure de $V_{R}$ étant de $10 \mathrm{~s}$ : ce rythme permet de suivre amplement les variations de résistivité de l'échantillon et donc la progression de l'hydrogénation. Remarquons également que la forte résistance de l'électrolyte qui est en parallèle de celle du ruban de $\mathrm{Pd}$, ne gêne pas la mesure de la résistance de ce dernier. Sur la figure 3 nous voyons une variation typique de la résistance $R$ du Pd en fonction du temps d'électrolyse $t: R$ croît d'abord avec $t$ (donc aussi avec $x$ ), passe par un maximum (pour $x \sim 0,75$ ) puis décroît; $R$ reprend la valeur $R(x=0)$ pour $x \simeq 0,97$ puis continue de décroître quand $x$ approche de l'unité. (Nous verrons plus loin comment nous mesurons $x$.) La durée de l'opération d'électrolyse est naturellement fonction de la densité de courant $j$ appliquée ainsi que de l'épaisseur des rubans de $\mathrm{Pd}$; nous avons observé que pour obtenir des concentrations voisines de $x \simeq 1$ il valait mieux utiliser des densités de courant $j$ de l'ordre de $1 \mathrm{~mA} / \mathrm{cm}^{2}$ et rallonger la durée d'électrolyse plutôt que d'essayer de forcer sur $j$ : des électrolyses par trop rapides provoquent en effet de forts gradients transitoires de concentration et donc de fortes contraintes internes et nous avons observé que l'hydrogène avait tendance à précipiter sous forme de bulles souvent visibles à l'œil nu; nous pensons que ces contraintes finissent par provoquer des fissures ou des cavités internes dans lesquelles l'hydrogène a tendance à s'accumuler; nous avons trouvé que ces précipitations étaient très sensibles à l'état métallurgique initial du $\mathrm{Pd}$, des échantillons bien recuits n'en présentant pratiquement pas. Nous avons observé également que pour des densités de courant trop élevées, il y avait dissolution progressive de l'anode de $\mathrm{Pt}$, suivie d'un dépôt de $\mathrm{Pt}$ sur le Pd; pour l'ensemble de ces raisons, il nous est apparu que des densités de courant supérieures à $60 \mathrm{~mA} / \mathrm{cm}^{2}$ devaient être proscrites.

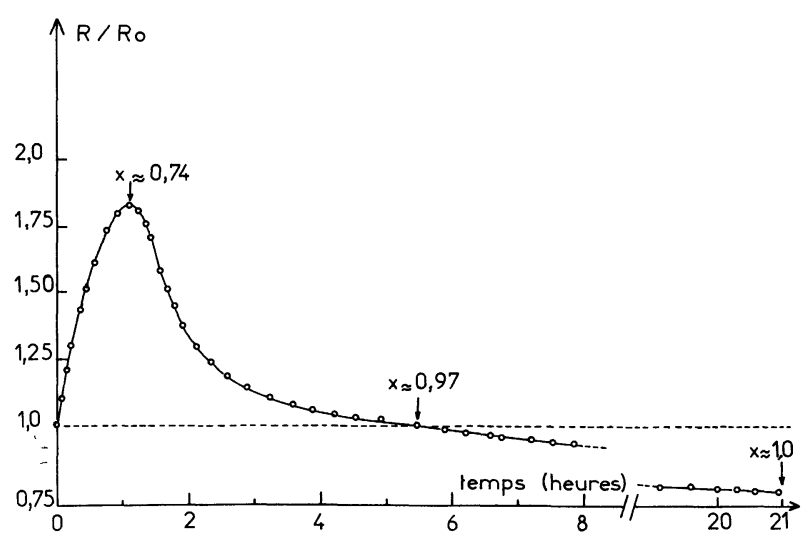

Fig. 3. - Résistance, normalisée à la valeur pour $x=0$, d'une lame de $\mathrm{Pd}$ en fonction du temps d'électrolyse, à la température de préparation de $195 \mathrm{~K}$.

[Normalized resistance of a Pd foil as a function of electrolysis time, at $195 \mathrm{~K}$.]
Une fois l'électrolyse terminée, le ruban est coupé au ras des deux tubes de verre, puis stocké dans l'azote liquide; toute manipulation ultérieure se fait autant que possible dans l'azote liquide, température à laquelle les échantillons sont parfaitement stables quel que soit $x$. En plus des mesures de résistivité, nous avons été capables de faire sur ces échantillons des mesures de susceptibilité magnétique, d'impédance de surface, de résonance électronique, d'effet tunnel..

3.1.4 Stabilité des hydrures concentrés $(x>0,7)$. - Les hydrures ainsi préparés sont d'un point de vue thermodynamique intrinsèquement instables, c'est-àdire qu'on devrait observer une désorption spontanée de l'hydrogène dès qu'on supprime la pression extérieure ou, dans notre cas, le courant d'électrolyse (tout comme un liquide s'évapore tant que la pression à sa surface est inférieure à sa pression de vapeur saturante). De fait, on observe que les hydrures massifs ainsi préparés sont parfaitement stables, même pour $x \simeq 1$, dans tout le domaine de température $T \lesssim-80^{\circ} \mathrm{C}$ (ceci n'est apparemment pas le cas pour les films minces qui manifestent de la désorption spontanée jusqu'à $-140^{\circ} \mathrm{C}$ [17]). $\mathrm{Si}$ on réchauffe un échantillon massif, on constate une désorption à partir d'environ - $60^{\circ} \mathrm{C}$ qui s'accélère très rapidement dès qu'on approche de la température ambiante; la désorption spontanée marque un arrêt aux alentours de $x \simeq 0,7$ et il faut chauffer les échantillons jusque vers $+300^{\circ} \mathrm{C}$ pour évacuer le reste de l'hydrogène ; ce comportement n'est pas bien compris à ce jour mais il marque une fois de plus la stabilité particulière liée à la concentration $x \simeq 0,7$; il est vraisemblable que des phénomènes de barrière de surface assurent la stabilité en dessous de $-80^{\circ} \mathrm{C}$.

3.1.5 Homogénéité des concentrations $x$. - Des gradients de concentration d'hydrogène peuvent s'établir aussi bien suivant l'épaisseur que suivant les autres dimensions de nos échantillons. Nous avons essayé de les estimer globalement en mesurant la largeur de transition supraconductrice $\Delta T_{\mathrm{c}}$ qui devrait être un paramètre sensible à ces gradients : la courbe $T_{\mathrm{c}}(x)$ présente au voisinage de $x \sim 0,95$ une pente de l'ordre de $0,7 \mathrm{~K} / \%$ de $x$ et de fortes variations de $x$ devraient se traduire par des transitions supraconductrices très élargies; les $\Delta T_{\mathrm{c}}$ observés n'ont jamais été supérieurs à environ $0,5 \mathrm{~K}$, d'où l'on peut déduire que $x$ est constant à environ $1 \%$ près sur tout le volume des échantillons. Remarquons également que l'absorption d'hydrogène est accompagnée d'une sensible dilatation du réseau; les gradients de concentration en hydrogène produisent donc des contraintes internes qui peuvent favoriser la formation de toutes sortes de défauts durant le processus de préparation. C'est pourquoi nous avons comparé la résistivité résiduelle d'un certain nombre d'échantillons avant hydrogénation et après désorption complète de l'hydrogène pour voir si un tel cycle hydrogénation-déshydro- 
génation introduisait de nouveaux défauts permanents. Nous n'avons en fait trouvé aucune variation importante et systématique de cette résistivité résiduelle.

3.1.6 Mesure de x. - Il n'est malheureusement pas possible de mesurer $x$ en intégrant le courant d'électrolyse car une bonne partie des ions $\mathrm{H}^{+}$ne pénètre pas dans le métal mais se dégage directement sous forme d'hydrogène moléculaire. L'évaluation de $x$ s'effectue donc, une fois toutes les mesures physiques faites, en mesurant le volume d'hydrogène désorbé après chauffage à $300^{\circ} \mathrm{C}:$ nous utilisons à cette fin un manomètre à mercure relié à une enceinte de volume constant; le $\mathrm{Pd}$ hydrogéné est introduit à froid dans cette enceinte, puis le vide y est fait, après quoi on laisse la température de l'enceinte remonter jusqu'à l'ambiante et on attend qu'un premier équilibre s'établisse. On effectue alors la mesure de la pression d'hydrogène ainsi dégagée ; pour extraire la totalité de l'hydrogène restant dans le métal, nous faisons à nouveau le vide dans l'enceinte et nous chauffons celle-ci à l'aide d'un four jusque vers $300^{\circ} \mathrm{C}$; de la somme des deux mesures de pression, nous déduisons la quantité $x$. Nous estimons la précision sur $x$ à mieux que $\pm 0,01$.

\section{Quelques résultats. - Nous allons décrire dans} ce dernier chapitre quelques résultats typiques qui illustrent l'intérêt de couvrir la gamme de concentration $0,7<x<1$, où apparaissent en fait nombre de propriétés nouvelles.

4.1 Résistivité et SUPRACONDUCTIVITÉ DE PdH PUR. - 4.1.1 Résistivité. - Nous venons de voir (Fig. 3) comment varie la résistivité électrique en fonction du temps d'hydrogénation, ceci à $-80^{\circ} \mathrm{C}$. La résistivité, à cette température, comporte des contributions venant aussi bien des défauts que des vibrations de phonons. Pour éliminer cette dernière contribution, nous avons effectué les mêmes mesures à $20 \mathrm{~K}$, cette fois en fonction de $x$ directement (Fig. 4).

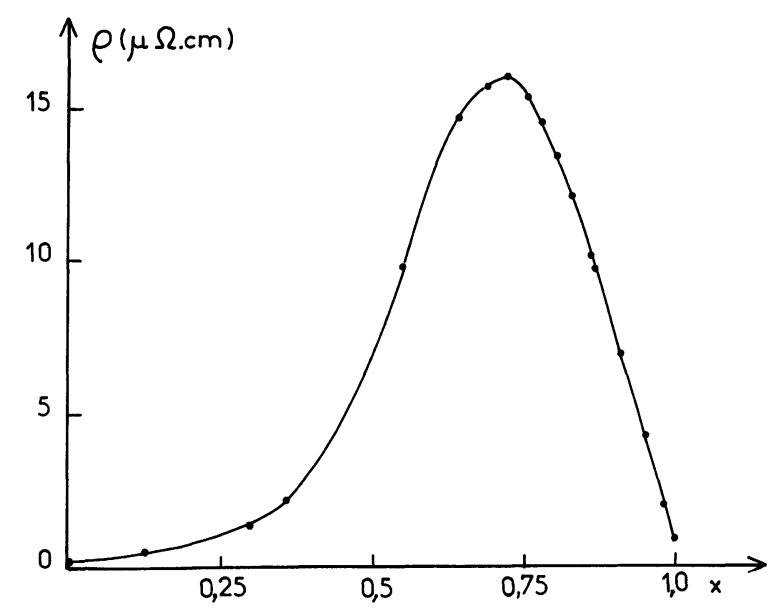

Fig. 4. - Résistivité résiduelle $\rho_{\mathrm{r}}$ d'une lame de Pd, mesurée à $20 \mathrm{~K}$ en fonction de la concentration d'hydrogène $x$.

[Residual resistivity of Pd, measured at $20 \mathrm{~K}$, as a function of $x$.]
Pour $x<0,7$ on observe une montée progressive de la résistivité résiduelle $\rho_{\mathrm{r}}$, manifestement due à la diffusion des électrons sur les atomes $\mathrm{H}$ jouant le rôle de défauts; cette montée ne nous renseigne pas sur la localisation de l'hydrogène dans le réseau du $\mathrm{Pd}$; pour $x>0,75$, on observe par contre une diminution rapide de $\rho_{\mathrm{r}}$ qui tend manifestement vers zéro quand $x$ tend vers 1 : ce résultat indique clairement que les protons se fixent dans les sites interstitiels octaédriques du Pd (il y a exactement un tel site par atome de Pd) et que la diffusion se fait sur les lacunes du réseau de l'hydrogène. Le composé stæchiométrique $\mathrm{PdH}$ finalement formé a donc la structure $\mathrm{NaCl}$. Nous n'avons observé aucune tendance vers l'occupation des sites tétraédriques, c'est-à-dire aucune tendance à former le composé $\mathrm{PdH}_{2}$, le rendement de l'électrolyse tendant invariablement vers zéro quand $x$ tend vers un. (Ce rendement se définit comme le rapport de la quantité d'hydrogène absorbée par le Pd à celle dégagée à l'électrode sous forme gazeuse.)

4.1.2 Supraconductivité. - Nous ne cherchons pas ici à présenter une étude exhaustive des propriétés supraconductrices de $\mathrm{PdH}_{x}$ observées récemment [18], mais nous nous contenterons de la mise en évidence d'un certain nombre d'effets liés directement à la qualité de nos échantillons.

Nous avons obtenu [15], dans la limite $x \rightarrow 1$, des températures de transition $T_{\mathrm{c}} \simeq 10 \mathrm{~K}$ supérieures d'au moins $1 \mathrm{~K}$ à celles obtenues, soit par pression, soit par implantation. Nous pensons que ceci est dû avant tout à l'obtention du composé stæchiométrique $\mathrm{PdH}$, clairement mise en évidence par le comportement de $\rho_{\mathrm{r}}$; nos résultats indiquent que

$$
\frac{\mathrm{d} T_{\mathrm{c}}}{\mathrm{d} x} \simeq 1 \mathrm{~K} / \% \text { de } x,
$$

valeur exceptionnellement élevée, qui montre l'extrême sensibilité de $T_{\mathrm{c}}$ à de faibles écarts à la stochiométrie ; le fait que nos $T_{\mathrm{c}}$ soient également supérieures à celles obtenues par implantation indique à notre avis que $T_{\mathrm{c}}$ est sensible de surcroît à l'ordre atomique et décroît pour des cristaux par trop perturbés.

Nous avons également mis en évidence [19] que $\mathrm{PdH}_{x}$ était un supraconducteur de première espèce pour $x>x_{\mathrm{c}}=0,98$ et devenait de $2^{\mathrm{e}}$ espèce pour $x<0,98$. Le passage $1^{\text {re }}$ espèce $\rightarrow 2^{\mathrm{e}}$ espèce est déterminé par la résistivité résiduelle qui est en première approximation proportionnelle au nombre de lacunes, c'est-à-dire $(1-x)$, ce dans la limite $x \simeq 1$. La valeur de $x_{\mathfrak{c}}$, très proche de l'unité, montre l'intérêt qu'il y a à bien contrôler les valeurs absolues de $x$, ainsi que la nécessité d'assurer une bonne homogénéité des concentrations en hydrogène.

Mentionnons également que des mesures de résistivité électrique en fonction de la température [20] ont permis de montrer que les vibrations des atomes $\mathrm{H}$ participaient à la diffusion des électrons et donc également à la supraconductivité. 
4.2 Hydrogénation D'alliages $\mathrm{Pd}_{1-y} \mathrm{H}_{y}$. - Nous avons étudié en détail l'influence de quelques impuretés $\mathrm{H}$ sur les concentrations maxima $x_{\mathrm{m}}$ d'hydrogène que peut absorber un alliage $\operatorname{Pd}_{1-y} \mathbf{M}_{y}$. On peut diviser ces impuretés en 2 catégories : d'une part, les impuretés $\mathrm{Ni}$ et $\mathrm{Pt}$ qui sont isoélectriques du Pd, et, d'autre part, les impuretés $\mathrm{Ag}, \mathrm{Fe}$, etc... qui sont situées respectivement à droite et à gauche de la colonne du Pd dans le tableau périodique des éléments. La figure 5 résume l'essentiel de nos résultats. Elle indique une faible variation de $x_{\mathrm{m}}$ pour les impuretés isoélectroniques, $x_{\mathrm{m}}$ restant voisin de l'unité, jusqu'à des concentrations de l'ordre de $10 \%$ en $\mathrm{M}$. On note ensuite une décroissance plus rapide pour Pt que pour $\mathrm{Ni}$, en accord avec le fait que Pt pur n'absorbe pas d'hydrogène. Pour les impuretés $\mathrm{Ag}$ et $\mathrm{Fe}$, on observe, par contre, une décroissance initiale beaucoup plus forte, ce qui indique à notre avis que ces impuretés substitutionnelles repoussent localement les atomes d'hydrogène; ceci devrait laisser de nombreux sites octaédriques vacants, un fait corroboré par l'étude de la résistivité électrique de ces alliages qui, pour la plupart, ne montrent plus de maximum de résistivité en fonction de $x$. Il n'est pas, a priori, facile d'expliquer les raisons de ce comportement mais on peut penser que la formation de la bande dite de l'hydrogène est perturbée par la présence des impuretés et que les électrons apportés par celles-ci participent au remplissage de la bande $\mathrm{d}$ du $\mathrm{Pd}$, déplaçant le niveau de Fermi vers le haut. Une étude plus poussée est manifestement nécessaire pour comprendre les effets systématiques produits par différentes impuretés sur la stabilité des hydrures. Remarquons simplement ici que les valeurs de $x_{\mathrm{m}}$ mesurées sont toujours supérieures d'au moins 20 à $30 \%$ à celles obtenues à température ambiante.

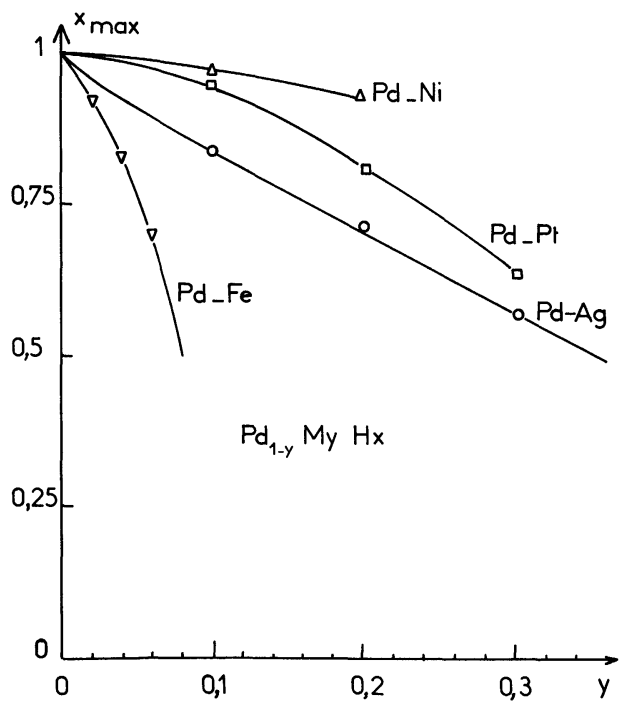

Fig. 5. - Concentration maximum $x_{\mathrm{m}}$ d'hydrogène absorbé à $195 \mathrm{~K}$ en fonction de la concentration d'impuretés substitutionnelles du Pd.

[Maximum concentration $x_{\mathrm{m}}$ of hydrogen absorbed at $195 \mathrm{~K}$ as a function of concentration of substitutional impurities.]
4.3 MagnÉtisme D'Alliages $\operatorname{Pd}_{1-y} \mathrm{M}_{y} \mathrm{H}_{x}$ - La plupart des impuretés magnétiques classiques $(\mathrm{Mn}$, $\mathrm{Fe}, \mathrm{Co}, \mathrm{Gd} . .$.$) forment de bons moments magnétiques$ aussi bien dans $\mathrm{Pd}$ que dans $\mathrm{PdH}_{x}$ [21]. Ces alliages doivent donc s'ordonner magnétiquement en dessous d'une température critique $T_{\mathrm{c}}$. Les interactions magnétiques entre impuretés responsables de cet ordre sont en général de nature indirecte et se font via la polarisation magnétique induite par chaque impureté dans la bande de conduction. On s'attend a priori à ce que ces interactions soient fortes et de longue portée dans le Pd pur qui possède une bande de conduction $\mathrm{d}$ à la limite d'être magnétique par ellemême, donc fortement polarisable; par contre la matrice $\mathrm{PdH}_{x}$ devrait conduire à des interactions normales telles qu'on les rencontre dans les métaux nobles monovalents usuels. Les observations que nous avons faites (Fig. 6) peuvent se résumer de la manière suivante :

- Les températures de transition $T_{\mathrm{c}}$ sont nettement plus petites (d'un facteur souvent supérieur à 10) dans les alliages hydrogénés que dans les mêmes alliages non hydrogénés [22].

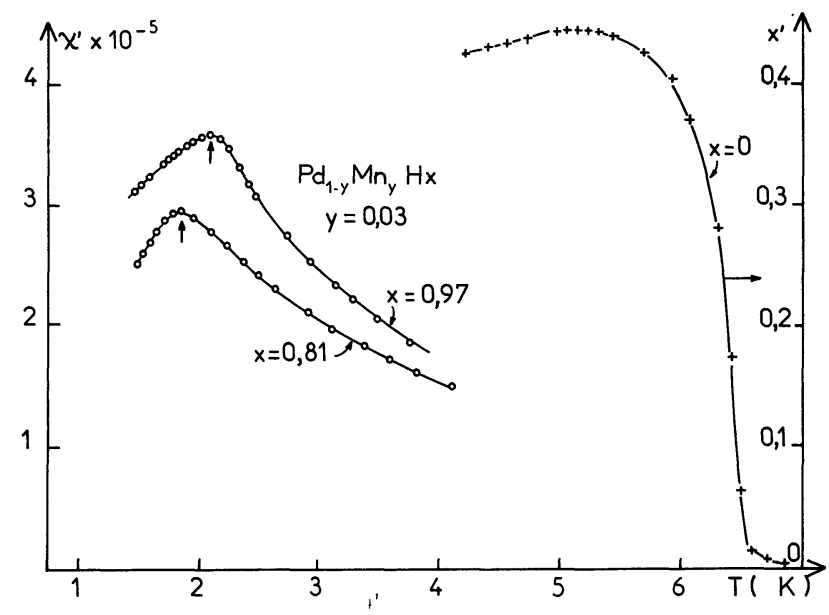

Fig. 6. - Susceptibilité en champ nul d'un alliage $\mathrm{Pd}_{1-y} \mathbf{M n}_{y} \mathrm{H}_{x}$ pour différentes valeurs de $x$. Notez la différence d'échelle entre $x=0$ (ferromagnétisme) et $x=0,81$ et 0,97 (verres de spin).

[Zero field magnetic susceptibility of an $\mathrm{Pd}_{1-y} \mathrm{Mn}_{y} \mathrm{H}_{x}$ alloy for different values of $x$. Notice the difference in scale for the $x=0$ (ferromagnetism) and $x=0.81$ and 0.97 (spin glasses) cases.]

- Il y a le plus souvent passage du ferromagnétisme pour les alliages non hydrogénés à un ordre magnétique du type verre de spin pour les alliages hydrogénés [23].

- Les températures $T_{\mathrm{c}}$ pour les alliages hydrogénés dépendent de la concentration $x$ (à concentration $y$ fixée). Cet effet est particulièrement intéressant dans la mesure où nous observons des corrélations entre les propriétés magnétiques et la densité d'électrons de conduction. 
5. Conclusion. - Nous avons mis au point une méthode simple et reproductible de préparation de composés $\mathbf{P d H}_{x}$ par électrolyse à basse température dans un bain d'alcool et d'acide sulfurique qui permet de couvrir la gamme de concentration $0,7<x<1$. Les composés les plus concentrés sont instables à température ambiante mais deviennent stables en dessous de la température de préparation de $-80^{\circ} \mathrm{C}$. Nous avons ainsi préparé pour la première fois le composé stœchiométrique $\mathrm{PdH}$ et montré que l'hydrogène occupe uniquement les sites interstitiels octaédriques dans le réseau du Pd. Nous avons montré sur plusieurs exemples l'intérêt et la nécessité qu'il y avait de s'approcher de la stœchiométrie vu la sensibilité de nombreuses propriétés (température de transitions supraconductrices, nature de la supraconductivité, résistivité résiduelle) à de faibles écarts à la stœchio- métrie. La possibilité de faire varier $x$ de manière continue entraîne une variation continue des propriétés électroniques (densité d'électrons de conduction, densité d'états au niveau de Fermi), ce qui conduit à d'intéressantes corrélations entre les propriétés magnétiques et $x$. Une étude plus exhaustive de la stabilité des hydrures les plus concentrés, en fonction de différentes impuretés. devrait conduire à une meilleure connaissance des facteurs qui gouvernent cette stabilité.

Remerciements. - Nous tenons à remercier Mme Boix qui a préparé et mis en forme nos échantillons; nous tenons à remercier de même $\mathrm{M}$. Le Pivert qui nous a aidés pour le montage des échantillons, le fonctionnement de l'électrolyse et le dépouillement des enregistrements.

\section{Bibliographie}

[1] Lewis, F. A., The Palladium-Hydrogen system (Academic Press) 1972

[2] Rowe, J. M., Rush, J. J., Smith, H. G., Mostoller, M., [3] Flotow, H. E., Phys. Rev. Lett. 33 (1964) 1297.

[4] Alefeld, G., Ber. Bunsenges. Phys. Chem. 76 (1972) 746.

[5] Switendick, A. C., Ber. Bunsenges. Phys. Chem. 76 (1972) 535.

[6] Mackliet, C. A., Schindler, A. I., Phys. Rev. 146 (1966) 463.

[7] Wucher, J., Ann. Phys. (Paris) 7 (1952) 317.

[8] Gupta, M., Freeman, A. J., Phys. Rev. 17 (1978) 3029.

[9] Eastman, D. E., Cashier, J. K., Switendick, A. C., Phys. Rev. 27 (1971) 35.

[10] Gelatt, C. D., Ehrenreich, H., Weiss, J., Phys. Rev. B 17 (1978) 1940.

[11] Baranowski, B. and Wisniewski, R., Phys. Status Solidi 35 (1969) 593.

[12] Skoskiewicz, T., Szafranski, A. W. and Baranowski, B., Phys. Status Solidi 59b (1973) K 135.

[13] Stritzker, B., Buckel, W., Z. Phys. 257 (1972) 1.
[14] Wicke, E., Nernst, G., Ber. Bun. Phys. Chem. 68 (1964) 224.

[15] Smith, R. J. and Otterson, D. A., J. Phys. Chem. Solids 31 (1970) 1187.

[16] HarPer, J. M. E., Phys. Lett. 47A (1974) 69.

[17] Arzoumanian, C., Thèse $3^{\mathrm{e}}$ cycle (Université d'Orsay, à paraître).

[18] Stritzker, B., WuHL, H., Topics in Applied Physics, Ed. Alefeld and Volk (Springer Verlag), (Hydrogen in Metals) 29 (1978) 243.

[19] Maclachlan, D. S., Doyle, T. B., Burger, J. P., J. Low Temp. Phys. 26 (1977) 589.

[20] Maclachlan, D. S., Mailfert, R., Burger, J. P., SoufFACHÉ, B., Solid. State Commun. 17 (1975) 281.

[21] Burger, J. P., Ann. Phys. (Paris) 9 (1964) 345.

[22] Burger, J. P., Senoussi, S., Souffaché, B., J. Less Common. Met. 49 (1976) 213.

[23] Burger, J. P., Maclachlan, D. S., Solid. State Commun. 13 (1973) 1563.

[24] Mueller, F. M., Freeman, A. J., Dimmock, J. O., FurDYNA, A. M., Phys. Rev. B 1 (1970) 4617. 